\title{
Research on Improved RSSI for Personal Positioning Method of Digital Community Monitoring System
}

\author{
Ke Hongdi ${ }^{1,}$ * * \\ 1 Department of Technology,Jilin Business and Technology College,Changchun 130507,China \\ a13661033@qq.coml
}

\begin{abstract}
Keywords: Digital Medical Care; Zigbee ;RSSI; Trilateral-weighted Centroid Localization Algorithm; Geometric Measurement Algorithm
\end{abstract}

\begin{abstract}
Zigbee network can be used to Digital Community Monitoring System, Personal Positioning Method is the important component of the Digital Community Monitoring System. On the basis of studying the RSSI and Trilateral-weighted centroid localization algorithms, we proposed a improved RSSI algorithm. On a wireless network hardware platform we validated that this geometric measurement algorithm had Higher precision than other algorithms.
\end{abstract}

\section{Introduction}

The development of community health service level represents the progress of a social level,correspondence the new medical technology such as modern network technology 、communication technology、 control technology and some medical equipment terminals, realize medical service and health care services, care services, emergency service, health education inside a family, build a digital community monitoring system is the the future of endowment trend. Construct digital community monitoring system, accurately master the position of the old man and monitor the health condition of the aged in real time[1,2].

Personal positioning method is the important component of the digital community monitoring system. The crucial of the Personal positioning method is to understand the health-status of the aged,and rescue them timely when they are in danger.

In this paper, based on the research object of the community aged, the positioning technology research is carried out to build the wireless network of the smart community medical guardianship. On the basis of studying the RSSI and Trilateral-weighted centroid localization algorithms, we proposed a improved RSSI algorithm[3,4].

\section{Positioning Algorithms}

Generally speaking, the distance independent positioning technology has low hardware requirements and low node cost, which is suitable for the positioning of large-scale wireless sensor network, but its positioning accuracy is not high. Distance based positioning technology is relatively high in hardware requirements and relatively high precision.

RSSI RSSI positioning technology is to estimate the distance between nodes by measuring the attenuation degree of the signal in the propagation process. The attenuation model of the node signal is represented by a logarithmic distance model[5,9].there is:

$$
\operatorname{PL}(\mathrm{d})=\operatorname{PL}\left(\mathrm{d}_{0}\right)-10 \mathrm{nlg}\left(\frac{\mathrm{d}}{\mathrm{d}_{0}}\right)-\mathrm{X}_{\sigma}
$$

$\mathrm{d}$-The distance between the launch node and the receiving node, $\mathrm{m} ; \mathrm{d} 0$ - reference distance, generally 1 $\mathrm{m} ; \mathrm{n}$-The attenuation index of the channel is an environment-related value, generally $2-4 ; \mathrm{X}_{\sigma-T h e}$ variance is $\delta$ gaussian random noise variable, the mean is $0 ; \operatorname{PL}\left(\mathrm{d}_{0}\right)$ - The signal intensity at $\mathrm{d} 0$ of the launch node can be obtained by experience or by the hardware specification, $\mathrm{dBm} ; \mathrm{PL}(\mathrm{d})$ - The intensity of the signal from the launch node $\mathrm{d}, \mathrm{dBm}$; 
For wireless sensor networks, RSSI algorithm is very environment-wide, and if no other modification methods are added, the error of positioning results may be large.

Trilateral-weighted Centroid Localization Algorithm. 3 known reference nodes A, B, C, blind node and the reference node distances are RA, RB, RC, centered on $\mathrm{A}, \mathrm{B}, \mathrm{C}, \mathrm{RA}$ and $\mathrm{RB}, \mathrm{RC}$ for the radius of circle. The intersection of three circle area, intersection of two circles set to $\mathrm{O} 1, \mathrm{O} 2, \mathrm{O} 3$ coordinates to satisfy the following equations[6,7]:

$$
\begin{aligned}
& x_{i}=\frac{x_{01} \cdot\left(\frac{1}{R_{B}+R_{C}}+\frac{1}{R_{A}^{n}}\right)+x_{02} \cdot\left(\frac{1}{R_{A}+R_{C}}+\frac{1}{R_{E}}\right)+x_{01} \cdot\left(\frac{1}{R_{A}+R_{B}}+\frac{1}{R_{C}}\right)}{\left(\frac{1}{R_{A}^{n}}+\frac{1}{R_{B}}+\frac{1}{R_{C}^{2}}\right)+\left(\frac{1}{R_{A}+R_{B}}+\frac{1}{R_{B}+R_{C}}+\frac{1}{R_{A}+R_{C}}\right)} \\
& y_{i}=\frac{y_{01} \cdot\left(\frac{1}{R_{B}+R_{C}}+\frac{1}{R_{A}^{n}}\right)+y_{0 x} \cdot\left(\frac{1}{R_{A}+R_{C}}+\frac{1}{R_{E}}\right)+y_{0:} \cdot\left(\frac{1}{R_{A}+R_{B}}+\frac{1}{R_{C}}\right)}{\left(\frac{1}{R_{R}^{n}}+\frac{1}{R_{B}}+\frac{1}{R_{R}}\right)+\left(\frac{1}{R_{A}+R_{B}}+\frac{1}{R_{E}+R_{C}}+\frac{1}{R_{A}+R_{C}}\right)}
\end{aligned}
$$

\section{Improved RSSI-- Geometric Measurement Algorithm}

Shown as Fig.1, Assume that there are 4 beacon nodes in a square field, and the location of the beacon node $\mathrm{kn} 1$ is the origin of coordinates, The distance between $\mathrm{kn} 1$ and $\mathrm{kn} 2 \mathrm{is} \mathrm{x}$, and the distance between $\mathrm{kn} 1$ and $\mathrm{kn} 4$ is regarded as the coordinate $\mathrm{y}$, which forms a node coordinate system, where D is the side length of the field. d1, d2, d3, d4, respectively, for the unknown node to four beacon node distance, if pending node within the field[8,9], so the location of the relationship between node as shown in Fig.1a, if the backlog nodes outside the venue, as shown in Fig.1b - 1e.

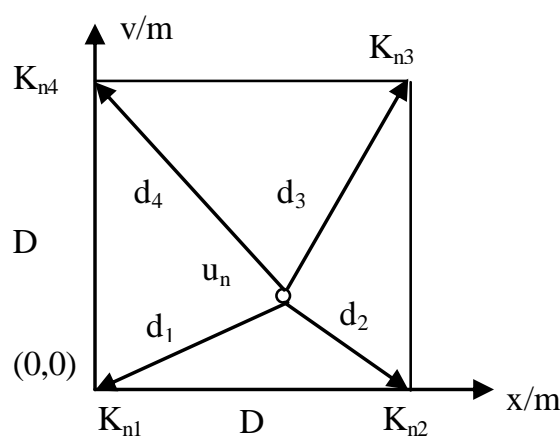

(a)

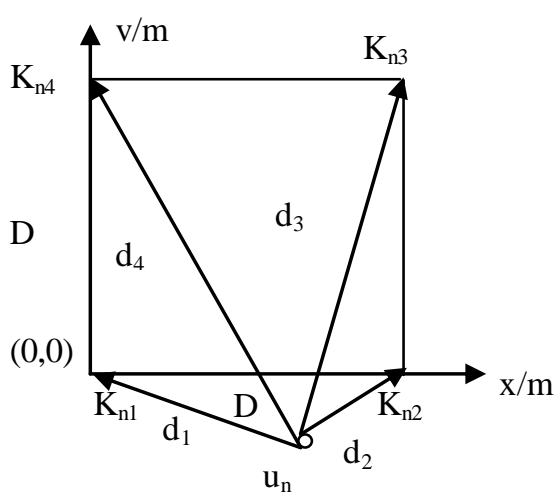

$(\mathrm{b}$

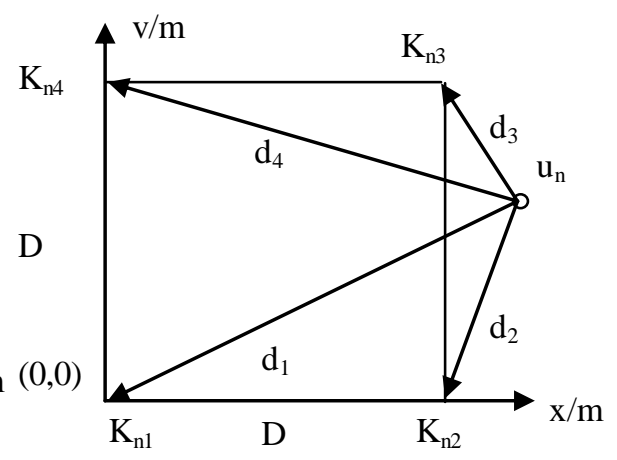

(c)

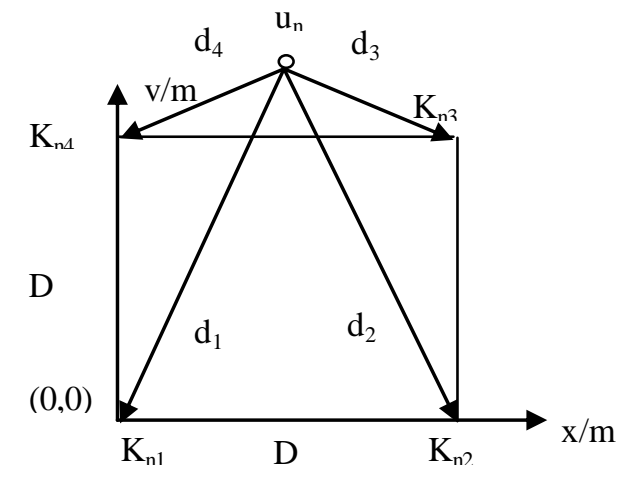

(d)

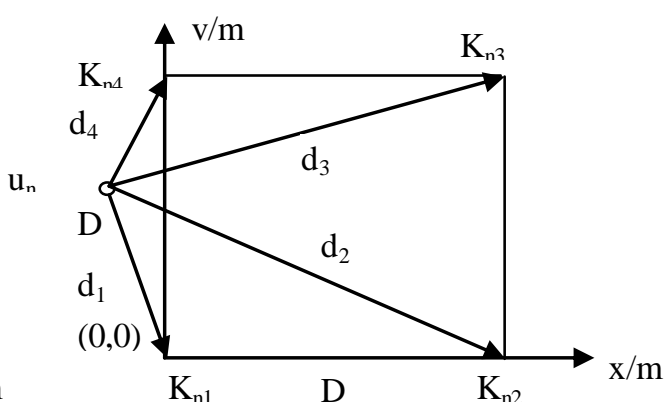

(e)

Figure 1. Location of beacon nodes and measurement node 
the geometric measurement algorithm can be:

$$
\begin{gathered}
\left\{\begin{array}{c}
x=\frac{D^{2}+d_{1}^{2}-d_{2}^{2}}{2 D} \\
y=-\frac{\sqrt{d_{1}^{2}-x^{2}}+\sqrt{d_{2}^{2}+(D-x)^{2}}}{2}
\end{array}\right. \\
\left\{\begin{array}{c}
y=\frac{D^{2}+d_{2}^{2}-d_{3}^{2}}{2 D} \\
x=D+\frac{\sqrt{d_{2}^{2}-y^{2}}+\sqrt{d_{3}^{2}+(D-y)^{2}}}{2}
\end{array}\right. \\
\left\{\begin{array}{l}
x=\frac{D^{2}+d_{4}^{2}-d_{3}^{2}}{2 D} \\
y=-\frac{\sqrt{d_{4}^{2}-x^{2}}+\sqrt{d_{3}^{2}+(D-x)^{2}}}{2} \\
x=D+\frac{\sqrt{d_{1}^{2}-y^{2}}+\sqrt{d_{4}^{2}+(D-y)^{2}}}{2}
\end{array}\right.
\end{gathered}
$$

\section{Matlab Simulate}

Beacon nodes are given respectively for 3 and 4,we compared the geometric algorithm error and trilateral-weighted centroid localization algorithm error, venue is $200 \mathrm{~m} * 200 \mathrm{~m}$, node communication distance is $100 \mathrm{~m}$, moves in a straight relationship between unknown nodes along the $\mathrm{y}=\mathrm{x}[10]$, evenly extraction sampling points, 51 can range in the path loss coefficient is 4.54 , The variance is 4.2 . from the simulation results of variance, as shown in the figure, you can see that the more sample point error is smaller.

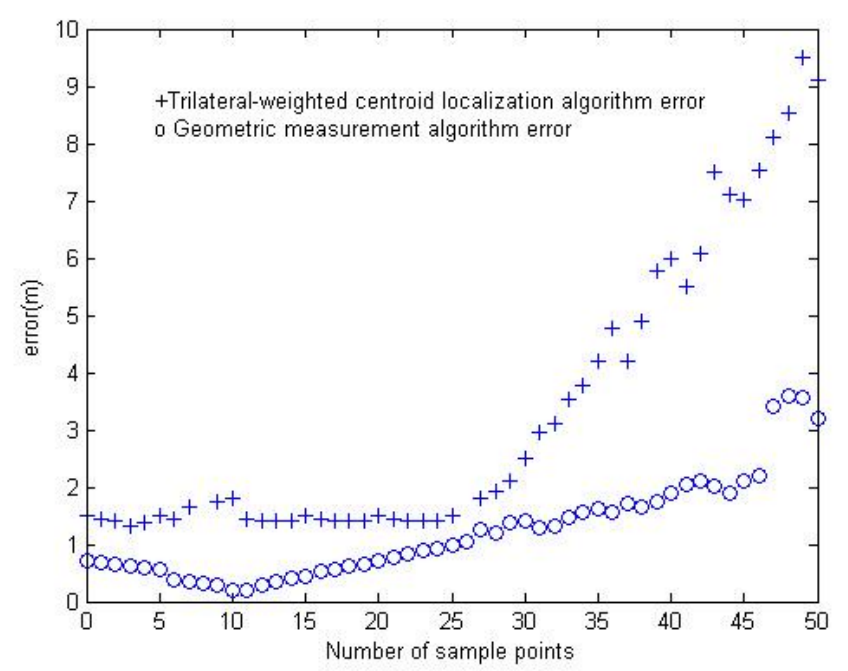

Figure 2. 4 beacon nodes 


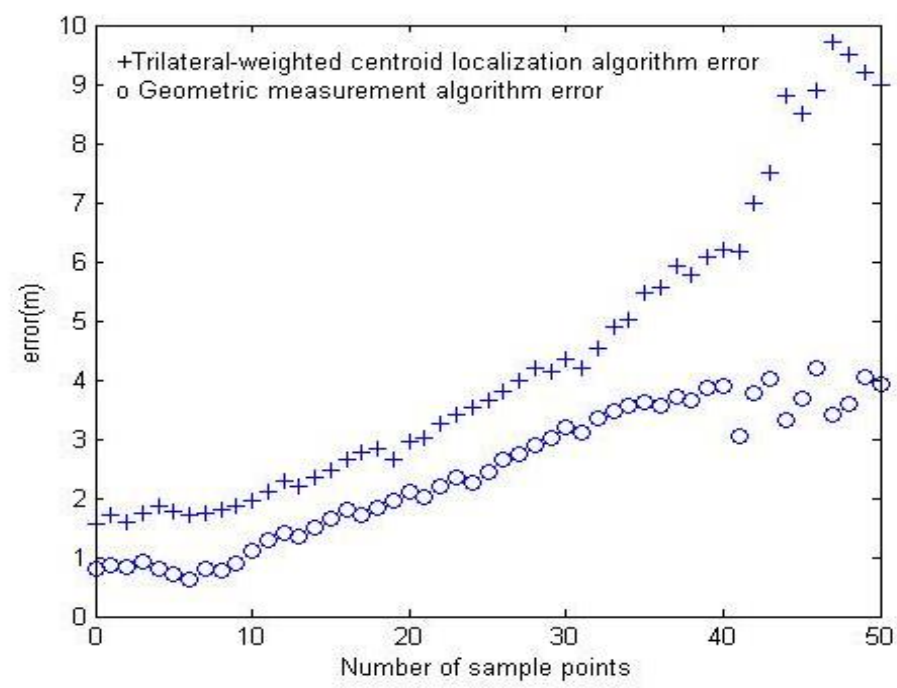

Figure 3.3 beacon nodes

\section{Summary}

In the topic, we proposed a improved RSSI algorithm. We used trilateral-weighted centroid localization algorithm and improved RSSI algorithm in Digital Community Monitoring System . On matlab simulated we validated that this algorithm had higher precision than traditional algorithms.

\section{Acknowledgements}

The research conducted in this paper have been supported by the fund of Jilin Provincial Education department(No.2014438) and JiLin Business and Technology doctor fund(No.201626).

\section{References}

[1] W.X.Tai:Research and Design of Mine Personnel Location System Based no Zigbee Wireless Sensor Network((Ms, Taiyuan University of Technology, China 2013), p.23.

[2] Mao Yurong: Control and Instruments in Chemical Industry, Vol.10.(2010).No.37,p91-94.

[3] Yang Wenbo,Xing Pengkang and Liu Yanhua:. Chinese Journal of Sensors and Actuators, Vol.28.(2015).No.1,p137-141.

[4] Zhou Wei: Research on Localization and Tracking Based on Wireless Sesor Networks((Ph.D., Chongqing Universityy, China 2011), p.65.

[5] Zheng Xueli,Fu Jingqi:Journal of Electronic Measurement and Instrument, Vol.36.(2015).No.5,p1177-1184.

[6] Zheng Xueli,Fu Jingqi:Chinese Journal of Scientific Instrument,Vol.36(2015).No.5, P1177-1184.

[7] Ding L,Guan X W:Foreign Electronic Measurement Technology.Vol.12(2014).No.33,P67-73.

[8]Tan Zhi,Zhang Hui: Journal of Beijing University of Posts and Telecommunications, Vol.36(2013).No.3,P88-91.

[9] Liu Chuanlai,Guo Lantian,Qin Haohua :Control and Instruments in Chemical Industry, Vol.39(2012).No.2,P204-208.

[10] Information on http://www.ilovematlab.cn/forum.php. 